\title{
Decentralized Control Strategy for PV Based DC Microgrid with Hybrid Storage
}

\author{
Ishita Biswas $^{1,}$, Prabodh Bajpai $^{1}$ \\ ${ }^{1}$ Department of Electrical Engineering, Indian Institute of Technology Kharagpur, 721302, West Bengal, India
}

\begin{abstract}
Due to the rapid development of power electronics technology and modernization of DC based loads, DC microgrids (DCMG) are becoming most attractive solution in terms of their increased efficiency and high power quality. However they face certain challenges like intermittency of renewable energy sources, limitations in dynamic behaviour of storage systems and various stability issues during islanding. Thus it is mandatory to select the DCMG components properly with a robust control strategy for power converters in order to maintain power balance despite of variation in source and load power in order maintain the system power quality. In this work, a DC bus signalling based decentralized control strategy is proposed for a PV based DCMG, which eliminates the risk associated with DC bus voltage fluctuation. Fuel cell is as an auxiliary power source along with solar PV and storage devices, reduces the possibility of black out and helps to increase system reliability. The proposed control strategy has been validated through MATLAB/Simulink based simulation studies for variable weather condition and load demand on DCMG system.
\end{abstract}

\section{Introduction}

Now a day's DC based power systems are becoming one of the most emerging solutions. However lots of challenges are associated with designing of DCMG system like stability of the system, effective control of DC bus voltage variation, optimal load sharing mechanism, paralleling of various distributed generation (DG) sources. A wide range of investigation, for configuration and control of DCMG with various combinations of renewable sources has already been carried out for both AC and DC based loads [1-4]. But the DGs with AC output increases power conversion steps. Also the use of $\mathrm{PV}$, wind both may cause problem in terms of higher system size, cost and unavailability of proper site. PV with battery as storage device is also becoming one of the most emerging structure, as PV has the natural DC coupling which makes it easier to connect to the common DC bus [5,6]. In addition to PV and Wind, Fuel cell (FC) is also becoming popular carbon free source due to high efficiency [7, 8]. Along with the DCMG system configuration, control of DG sources is another important aspect which is an emerging area of research in recent years. Hierarchical control approaches associated with ACMG can be applied to DCMG system also, which is mainly divided into three categories as primary, secondary and tertiary control [9, 10]. Issues of poor voltage regulation and circulating current among DG sources [11] can be avoided in case of secondary control which is mainly based on

\footnotetext{
${ }^{a}$ Corresponding author : i.biswas4@gmail.com 
energy management strategy. Secondary control is of two types, centralized and decentralized. Centralized controller mainly mitigates the voltage imbalance caused by primary control, but it needs a real time communication system for operation. Decentralized control can be modelled with or without communication system. Thus the communication problem can be eliminated with DC bus signalling technique [12].

In this paper, a PV based DCMG system with hybrid energy storage system (HESS), comprising of battery and SC both is considered for study. In addition, a FC is considered for the emergency condition to minimize load shedding. A DC bus signalling based decentralized control algorithm is formulated for this DCMG system where the DC bus voltage is used as the key parameter to decide the system operating modes and the control signals for local controllers are computed.

\section{Structure of DCMG and operating modes}

The proposed DCMG system considering PV-FC-Battery-SC is shown in Fig. 1(a). The system component sizing is based on optimal results of PSO-EO heuristic method [13]. The sizing details are not covered here due to space limitations. It consists of main source, solar PV $(520 \mathrm{kWp})$ connected to the DC bus with DC-DC buck converter-I. The controlled source of energy, utility grid, is connected to the DC bus by grid connected voltage source converter (G-VSC-I) $(500 \mathrm{~kW})$ and a static transfer switch (STS), an intelligent bypass switch (Normally closed) to continuously monitor the mode of operation of DCMG [14]. SC bank $(63 \mathrm{~F} \times 5)$ and Battery $(5 \mathrm{kAh}, 400 \mathrm{~V})$ are used as HESS. Two bidirectional DC-DC converters (converter II and III) connect HESS elements to DC bus. FC (450kW) is interfaced with DC bus by means of DC-DC boost converter-IV. A combination of one constant AC load-1 $(150 \mathrm{~kW})$ and three variable DC load 2, 3, $4(125 \mathrm{~kW} \times 3)$ is considered. DCMG operation is broadly classified in two modes, depending on the grid connection condition with DCMG [15] as follows:

\subsection{Mode-1/Grid connected (GC) mode}

This mode corresponds to the DCMG operation in connection with utility grid. In this mode, Solar PV always delivers maximum power $\left(P_{p v}\right)$ and storage devices (SC and Battery) can take charging/ standby power $\left(P_{s c}, P_{b a t}\right)$ to attain their maximum SoC level. Any surplus or deficit power within the DCMG is balanced by grid power $\left(P_{g}\right)$ by power control mechanism of G-VSC-I as presented in equ.1, where $P_{\text {load }}$ is the load power $[16,17]$.

$$
P_{p v}+P_{g}=\left(P_{\text {load }}+P_{s c}+P_{b a t}\right)
$$

\subsection{Mode-2/Standalone (SA) mode}

In this mode, PV operates at MPPT, SC power $\left(P_{s c}\right)$ maintains the DC bus voltage, battery maintains a certain $\mathrm{SC}$ voltage level by supplying required charging/discharging power to the system according to equ.2. In this work, FC supplies the load and charges the HESS in case of lower battery SoC level. FC power $\left(P_{f c}\right)$ maintains system power balance as expressed in equ.3, even if PV power and HESS are not available enough to support.

$$
\begin{gathered}
P_{p v}+P_{b a t}=\left(P_{\text {load }}+P_{s c}\right), \text { when } \mathrm{FC} \text { is OFF } \\
P_{p v}+P_{f c}=\left(P_{\text {load }}+P_{s c}+P_{b a t}\right), \text { when FC is ON }
\end{gathered}
$$

\section{Power management strategy for DCMG system}


Two possible scenarios are considered as the cause of grid outage. One is a short period AC grid fault, which results in grid side AC voltage dip and reduction of power transfer limit of G-VSC-I. The second possibility can be collapse of grid or grid isolation due to maintenance purpose. In both situations, G-VSC-I becomes incapable of maintaining system power balance. Thus HESS must control the system and system switches to SA mode. The flowchart presented in Fig.1 (b) describes the changeover logic with power management strategy.

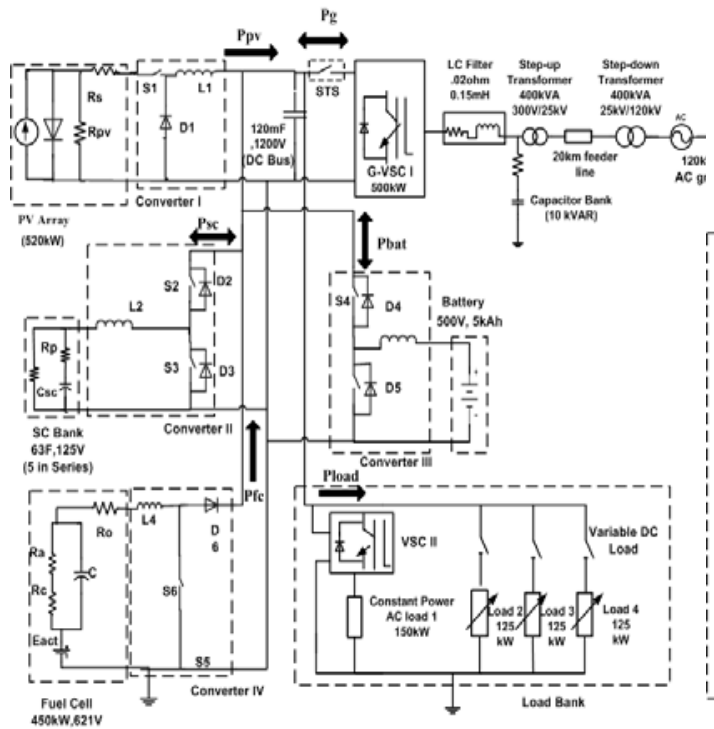

(a)

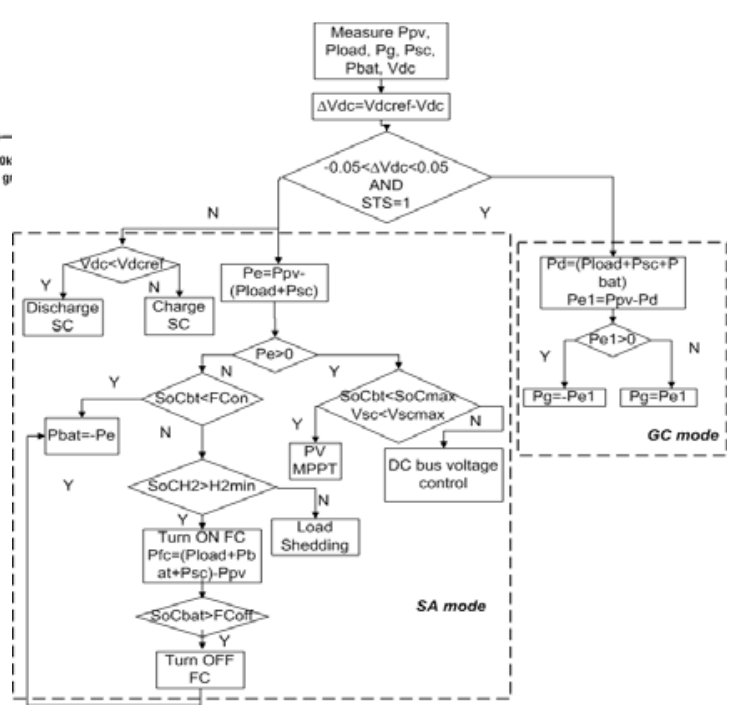

(b)

Figure 1. (a)Structure of proposed DCMG with system rating, (b) Changeover logic for DCMG system

The algorithm initiates with the measurement of PV power $\left(P_{p v}\right)$, load power $\left(P_{l o a d}\right)$, battery power $\left(P_{b a t}\right)$, SC power $\left(P_{s c}\right)$, grid power $\left(P_{g}\right)$, DC bus voltage $\left(V_{d c}\right)$ and the state of STS $(0 / 1)$. The DC bus voltage $\left(V_{d c}\right)$ and STS state determine the mode of operation. In GC mode, when bus voltage lies within $\pm 5 \%$ and STS is closed, the excess/deficit PV power is balanced by grid, in order to supply the load and to maintain maximum storage level. But, if the bus voltage violets its regulation range and STS also gets opened due to the detection of AC fault/grid failure, the system operation shifts to SA mode. In SA mode, HESS plays key role to maintain power balance, where SC maintains DC bus voltage by charging/discharging during transient load condition and battery takes care of steady state load. In SA mode, the excess PV power $\left(P_{e}\right)$ charges the battery and SC, with MPPT operation, however, if HESS is fully charged, MPPT operation is suspended. If $P_{e}<0$, battery first takes the charge of load, until it discharged up to a certain SoC level, where FC operation is scheduled. At this level FC turns ON and supplies the load, and charge HESS up to maximum SoC level. As soon as HESS gets charged, FC turns OFF and system operation continues with PV, SC and battery only.

\section{Simulation results and discussions}

The proposed control strategy has been validated for GC and SA modes of operation of hybrid storage DCMG system through simulation studies.

\subsection{Normal GC operation}

The proposed hybrid storage DCMG control strategy has been validated for GC mode with desired power sharing as shown in Fig.2. PV power is varying with solar irradiance, but load end DC bus voltage is maintained constant. 
Fig.2 (a) illustrates power sharing among the PV, grid, HESS and load grid across the DC bus. In Part-1 and 2, grid exports power to DCMG to maintain power balance, since PV power is not enough to supply the load as well as fully charge the HESS. In part III, $P_{g}$ approaches to zero and start importing power from DCMG as $P_{p v}>P_{\text {load }}$ and HESS is almost fully charged. The PV power decreases, but load demand has increased in Part 4 (1.57-2.5sec), so grid again starts exporting power to DCMG. Fig. 2 (b), (c), (d) and (e) are showing the variation in solar irradiance, SC voltage, DC bus voltage and battery SoC level respectively.
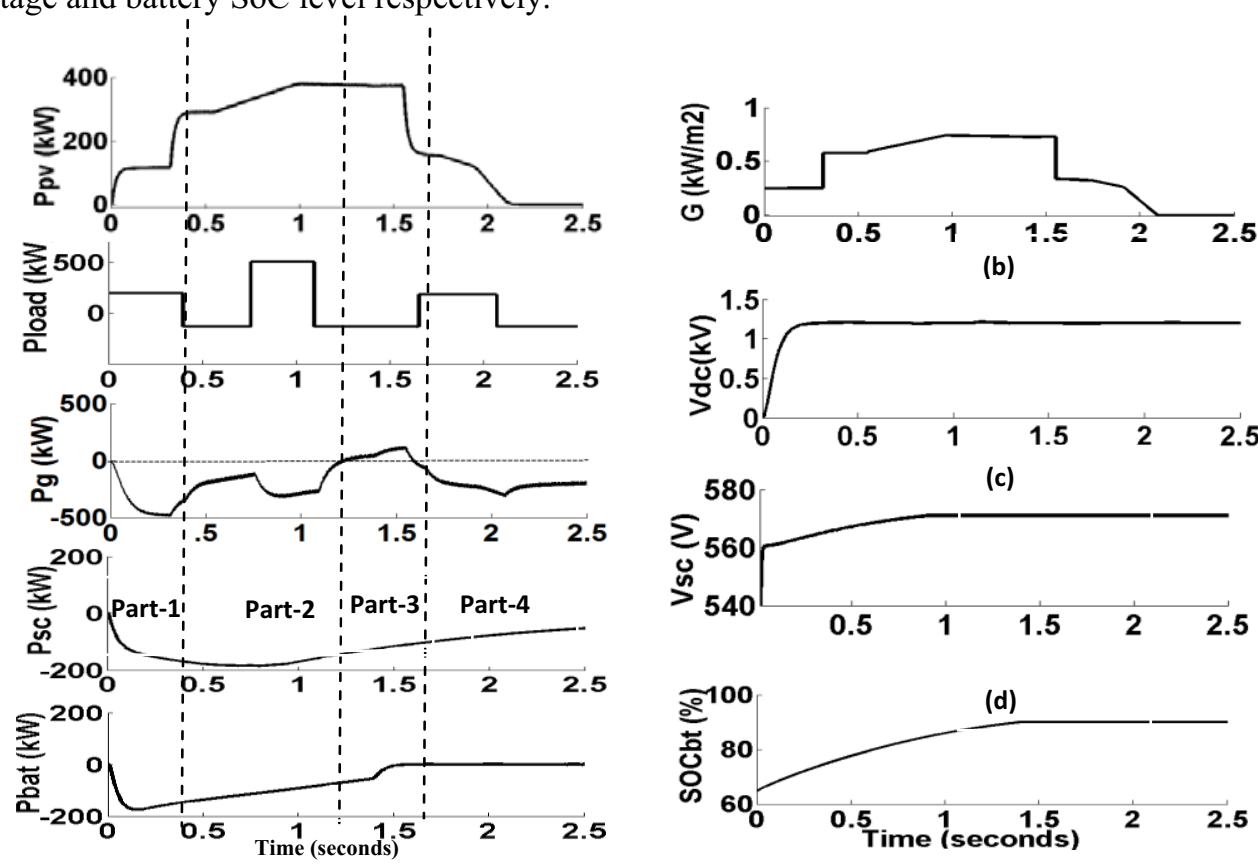

(b)
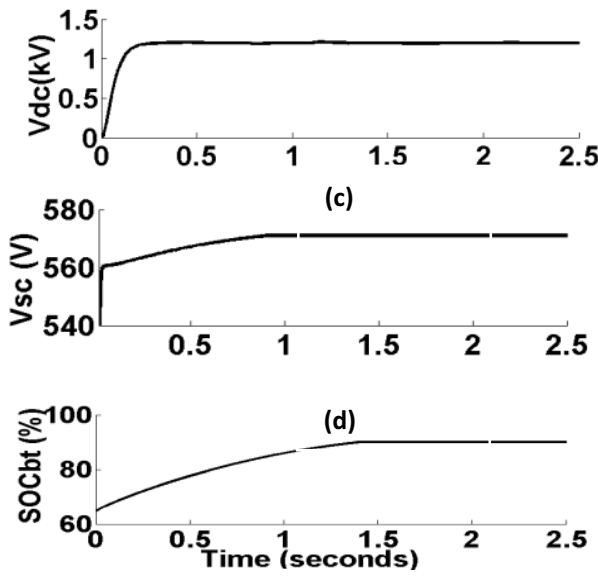

(a)

(e)

Figure 2 . (a) Power sharing strategy across DC bus among sources, HESS and load in GC mode(b) Solar irradiance profile (c) DC bus voltage (d) SC voltage (e) Battery SoC (\%)

\subsection{Impact of AC fault / grid collapse}

Changeover operation during AC fault followed by grid collapse is described in this section through various plots in Fig.3. The power plots presented in Fig.3 (a) is divided into four parts. In Part-1, grid exports power to DCMG to maintain power balance in the system. Part-2 $(0.5-0.7 \mathrm{sec})$ simulates a short duration single line to ground fault at the end of transmission line on grid side, at $0.5 \mathrm{sec} . \mathrm{Pg}$ approaches to zero as STS detects the fault. So SC discharges to maintain $V_{d c}$ constant (at $\left.1.2 \mathrm{kV}\right)$ and battery charge SC for voltage control of SC. In Part-3 $(0.7-1.5 \mathrm{sec})$, as soon as the fault gets cleared at $0.7 \mathrm{sec}$, the system moves back to GC mode, and due to low PV power and insufficient storage level, grid again starts exporting power to DCMG (Pg is negative). Grid gets disconnected again at $1.5 \mathrm{sec}$ in Part-4 due to grid collapse and Pg approaches to zero value. STS detects grid failure and HESS supply the load with partial support from PV. Fig.3(c) shows that $V_{d c}$ is maintained constant during changeover from GC to SA mode and back.

\subsection{Standalone operation}

DCMG operation in SA mode based on proposed power management strategy is validated in this section considering FC as auxiliary power source. For this simulation, FC ON and FC OFF levels are chosen as $50 \%$ and $90 \%$ of battery SoC level respectively. Fig.4 (a) is illustrating power sharing among sources and load in three different parts. Now $P_{g}$ plot is replaced with $P_{f c}$ plot from previous section. In the Part- $1, P_{p v}$ is less that $P_{\text {load }}$, so battery and SC support to meet the load and maintain 
DC bus voltage constant. In Part-2, $P_{p v}$ is zero and SoCbt discharges to $50 \%$ as shown in Fig.4 (e). Thus FC gets $\mathrm{ON}$ at $0.85 \mathrm{sec}$, and starts supplying a constant power of $450 \mathrm{~kW}$. In Part-3, FC turns OFF at $11 \mathrm{sec}$ when battery SoC reaches $90 \%$ SoC. So battery and SC meet the load and $1.86 \mathrm{sec}$. PV power is available so it starts sharing the load power with battery. But Ppv and Pbat are together enough for load demand so SC starts charging with $P_{s c}$ in negative region.
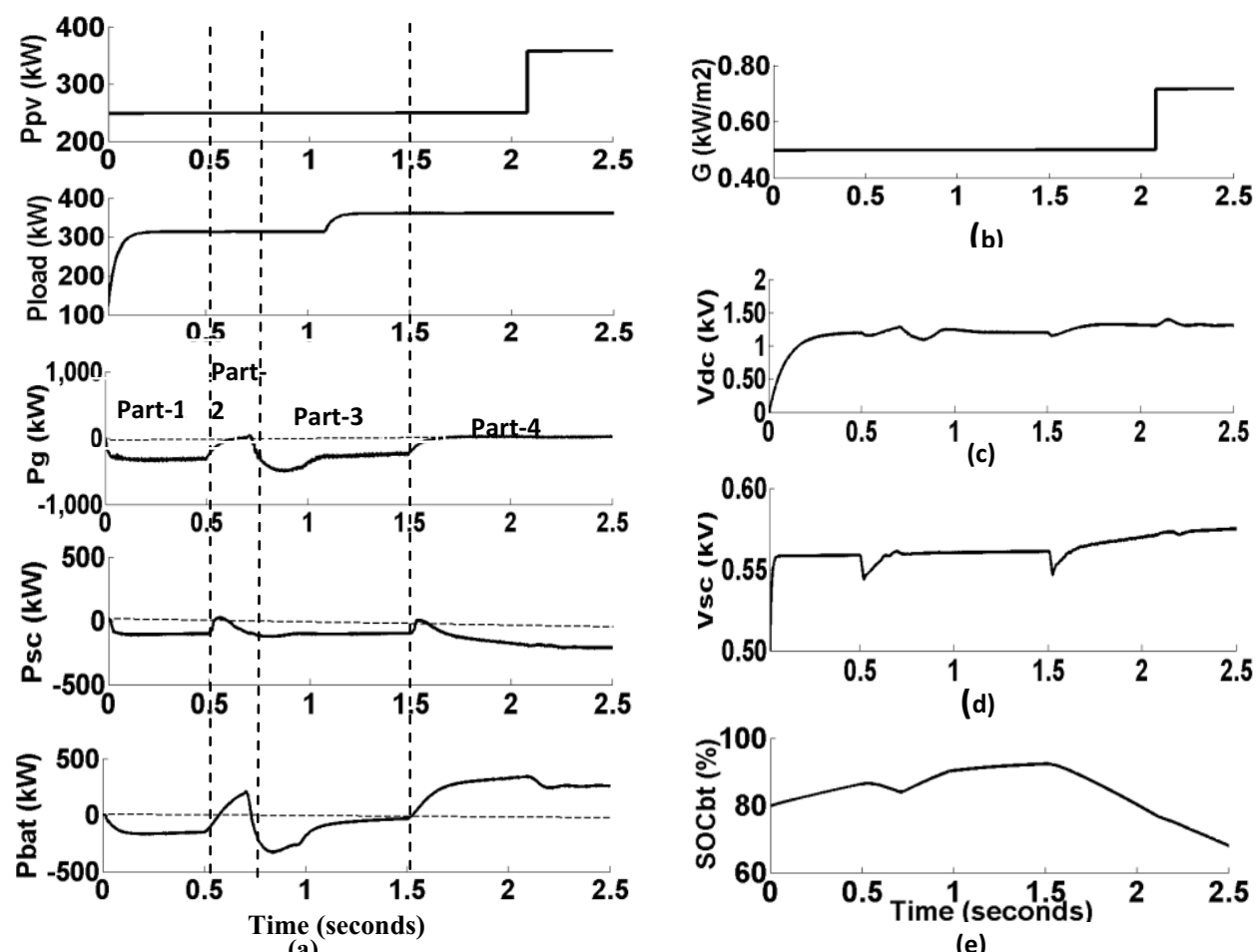

(e)

Figure 3. (a) Power sharing strategy across DC bus among sources, HESS and load in case of AC fault/grid collapse (b) Solar irradiance profile (c) DC bus voltage (d) SC voltage (e) Battery SoC (\%)

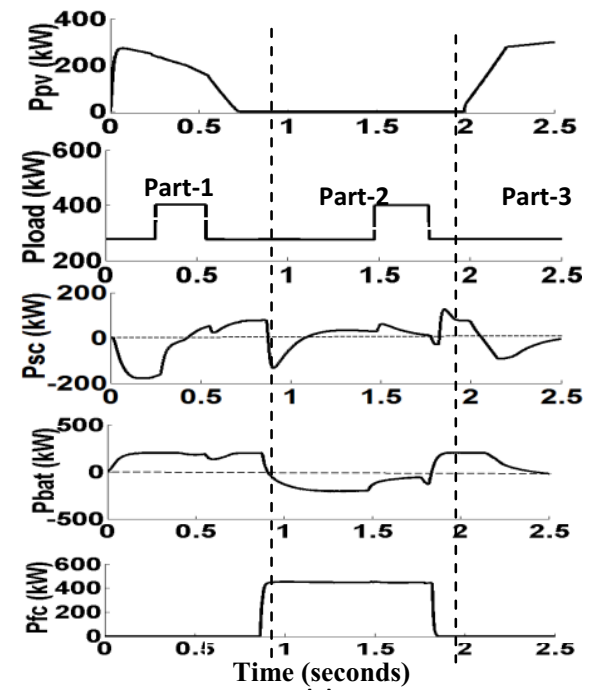

(a)
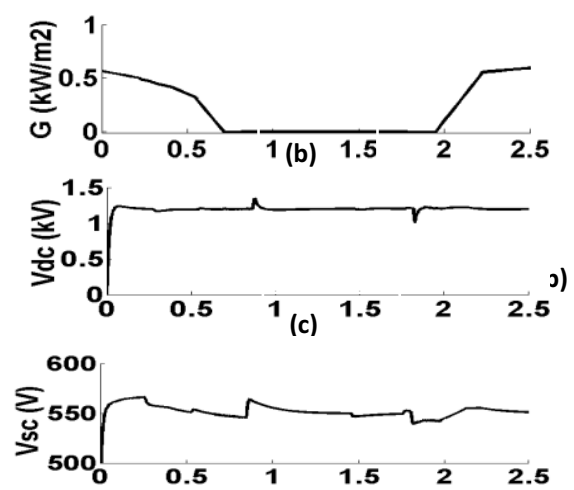

(d)

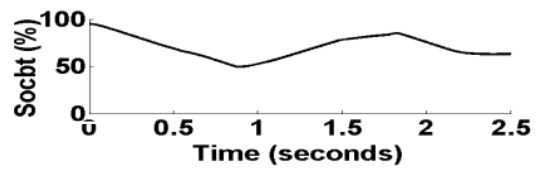

(e)

Figure 4. (a) Power sharing strategy across DC bus among sources, HESS and load in case of SA mode (b) Solar irradiance profile (c) DC bus voltage (d) SC voltage (e) Battery SoC (\%) 


\section{Conclusion}

In this paper, a DCMG system is designed to get high reliability in terms of uninterruptable power supply and the quality of power is maintained by always limiting load end voltage variation. A decentralized control strategy to maintain constant DC bus voltage of the DCMG is validated with various simulation studies under GC mode, SA mode and changeover between them. The desired performance is achieved with proposed HESS in DCMG and FC as auxiliary power source.

\section{Acknowledgement}

The authors are thankful to the authority of Electrical Engineering department, IIT, Kharagpur and VICET, for their encouragement and support for this work.

\section{References}

1. A. Khorsandi, M. Ashourloo, H. Mokhtari, A Decentralized Control Method for a Low-Voltage DC Microgrid, IEEE Trans. Energy conversion, 29(4), 793-801(2014)

2. D. Chen, L. Xu, Autonomous DC voltage control of a DC microgrid with multiple slack terminals,IEEE Trans. Power System. 27(4), 1897-1905 (2012)

3. L. Xu, D. Chen, Control and design of a DC microgrid with variable generation and energy storage, IEEE Trans. on Power Delivery, 26(4), 2513-2512 (2011)

4. J. Guerrero, J. Vasquez, J. Matas, M. Castilla, L. de Vicuna, Control strategy for flexible microgrid based on parallel line-interactive ups systems, IEEE Trans. Ind. Electronics, 56(3), 726-736 (2009)

5. X. Wang, M. Yue, E. Muljadi, Modelling and control system design for an integrated solar generation and energy storage system with a ride-through capability, ECCE, 3727-3734 (2012)

6. S.K. Kim, J.-H. Jeon, C.-H. Cho, J.-B. Ahn, Modelling and simulation of a grid connected PV generation system for electromagnetic transient analysis, Solar Energy, 83, 664-678 (2009)

7. M. Mohammadi, M. Nafar, Fuzzy sliding-mode based control (FSMC) approach of hybrid microgrid in power distribution systems, J. Elect Power and Energy System, 51, 232-242 (2013)

8. A. Ghazanfari, M. Hamzeh, H. Mokhtari, H. Karimi,Active power management of multihybrid fuel cell/supercapacitor power conversion system in a medium voltage microgrid, IEEE Trans. on Smart Grid, 3(4), 1903-1910 (2012)

9. J.M. Guerrero, J.C. Vasquez, J. Matas, L.G. de Vicũna, M. Castilla, Hierarchicalcontrol of droopcontrolled AC and DC microgrids - a general approach toward standardization, IEEE Trans. Ind. Electron. 58 (1), 158-172 (2011)

10. S. Anand, B.G. Fernandes, M. Guerrero, Distributed control to ensure proportional load sharing and improve voltage regulation in low voltage DC microgrids, IEEE Trans. Power Electron. 28 (4), 1900-1913(2013)

11. C. N. Papadimitriou, E. I. Zountouridou, N. D. Hatziargyriou, Review of hierarchical control in DC microgrids, Electric Power Systems Research, 122 (0), 159-167 (2015)

12. J. Bryan, R. Duke, S. Round, Decentralized generator scheduling in a nanogrid using DC bus signalling, IEEE PES GEM. 977-982(2004)

13. Biswas, I, Dash. V, Bajpai, P, Sizing optimization of PV-FC-Battery system with hybrid PSO-EO algorithm," in India Conference (INDICON), 2012 Annual IEEE, 869-874, 2012.

14. X. Wang, M. Yue, E. Muljadi, Modelling and control system design for an integrated solar generation and energy storage system with a ride-through capability, ECCE, 3727-3734 (2012)

15. N. Eghtedarpour E. Farjah, Power control and management in a hybrid AC/DC microgrid, IEEE Trans. Smart Grid, 5(3), 1494-1505 (2014)

16. E. Serban, H. Serban, A control strategy for a distributed microgrid application with voltage \& current-controlled source converter, IEEE Trans. on Power Elect., 25(12), 2981-2992 (2010)

17. S. R. Nandurkar, M. Rajeev, Design and Simulation of three phase Inverter for grid connected Photovoltaic systems, IEEE APECE, 80-83 (2012) 